\title{
Surveys in Surgical Education: A Systematic Review and Reporting Guideline
}

\author{
Louise B.D. Banning ${ }^{a} \quad$ Vincent M. Meyer ${ }^{b} \quad$ Joost Keupers ${ }^{a} \quad$ Johan F.M. Lange ${ }^{a}$ \\ Robert A. Pol ${ }^{\mathrm{a}}$ Stan Benjamens ${ }^{\mathrm{a}}$ \\ a Department of Surgery, University of Groningen, University Medical Center Groningen, Groningen,

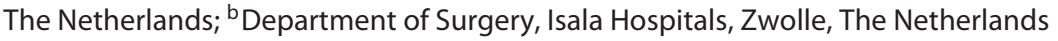

\section{Keywords}

Surveys and questionnaires - Systematic review ·

Education · General surgery

\begin{abstract}
Objectives: Survey studies are a commonly used method for data collection in surgical education research. Nevertheless, studies investigating survey design and response rates in surgical education research are lacking. The aim of this study was to gain an insight into survey response rates among surgical residents and medical students, and provide an initial reporting guideline for future survey studies in this field. Design: PubMed (MEDLINE) was systematically searched for survey studies in surgical education from January 2007 until February 2020, according to the PRISMA statements checklist. Study selection was conducted by 2 authors, independently. Surveys directed at surgical residents and/or medical students were included if data on response rates was available. Studies reporting solely from nonsurgical fields of medicine, paramedicine, or nursing were excluded. Subgroup analyses were performed, comparing response rates for varying modes of survey, per country, and for the 10 journals
\end{abstract}

with the most identified surveys. Results: From the 5,693 records screened for a larger surgical survey database, a total of 312 surveys were included; 173 studies focused on surgical residents and 139 on medical students. The mean (SD) response rate was $55.7 \%(24.7 \%)$ for surgical residents and $69.0 \%(20.8 \%)$ for medical students. The number of published surveys increased yearly, mostly driven by an increase in surgical resident surveys. Although most surveys were Web-based ( $n=166,53.2 \%$ ), this survey mode resulted in the lowest response rates (mean 52.6\%). The highest response rates, with a mean of $79.8 \%$ (13.1\%), were seen in in-person surveys ( $n=89,28.5 \%$ ). Wide variations in response rates were seen between different countries and journals. Conclusions: Web-based surveys are gaining popularity for medical research in general and for surgical education specifically; however, this mode results in lower response rates than those of in-person surveys. The response rate of in-person surveys is especially high when focusing on medical students. To improve reporting of survey studies, we present the first step towards a reporting guideline.

(C) 2021 The Author(s)

Published by S. Karger AG, Basel
(C) 2021 The Author(s)

Published by S. Karger AG, Basel

This is an Open Access article licensed under the Creative Common Attribution-NonCommercial-4.0 International License (CC BY-NC) (http://www.karger.com/Services/OpenAccessLicense), applicable to the online version of the article only. Usage and distribution for commercial purposes requires written permission.
Correspondence to:

Stan Benjamens, s.benjamens@umcg.nl 


\section{Introduction}

Survey studies are frequently applied for the evaluation of research into education for surgical residents and medical students [1]. These surveys form a key element for improving medical education and assessing the performance, attitudes, and well-being of the residents and students.

Surveys can be conducted in modes such as in-person interviews, postal questionnaires, and Web-based surveys [2], the last of which has gained popularity in recent years [3]. It has been well established that different survey types will produce a variable response rate, which, in turn, affects their reliability and interpretation. A low response rate introduces a nonresponse bias and wider confidence intervals, thereby affecting the quality of conclusions drawn from a survey [4]. In-person surveys show higher response rates than studies with a postal and/or Webbased design but are more expensive to perform. Webbased surveys, on the other hand, are easy to implement and more suitable for a large sample size, but response rates are relatively low $[5,6]$. Specific to medical students, researchers found a $50 \%$ drop in the response rate when a postal survey was replaced by an e-mail-based survey [7].

Although medical students are frequently approached for surveys, little is known about their rate of participation [8-10]. Medical students and residents are subject to the hierarchy at medical school and can react and respond to survey requests differently from other health care professionals [11]. It is thus difficult to compare their participation rates. A study that analyzes the rate of participation by this specific group in different survey types is needed to maintain reliable survey results. However, studies investigating the survey methods and response rates in surgical education research are currently lacking [12]. Analyzing the participation in surveys and the survey methods in specific populations, e.g., medical students and residents, can provide essential information to improve the quality of future surveys and survey research in general [5].

Survey research is often hindered by unclear and inconsistent reporting of the methods used, e.g., the response rates and the number of survey requests $[10,13]$. This lack of information about survey methodology hampers the reliability and reproducibility of the results. Therefore, the implementation of a standardized reporting guideline for surveys in surgical education is needed. The aim of our study was to perform a systematic review on surveys in surgical education, with an emphasis on response rates for varying modes of survey, and to present an initial reporting guideline for future studies.

\section{Methods}

PubMed (MEDLINE) was systematically searched from 1 January 2007 to 1 February 2020, resulting in the surgical survey database referred to by Meyer et al. [2] in the Annals of Surgery. For this database, the following MeSH term or keywords were used: ("questionnaire" or "survey") and "response rate" and ("surgery" or "surgical"). A subgroup of surgical resident and medical student surveys was selected by searching for "resident," "trainee," "student," or "intern". Studies were included if matching criteria for surgical resident or medical student and data on response rates were available. Reviews, conference abstracts, case reports, and studies reporting solely from nonsurgical fields of medicine, paramedicine, or nursing, were excluded. Studies reporting the use of various survey types were also excluded. Two authors (L.B.D. and S.B.) independently carried out the study selection based on the stated criteria. This systematic review was performed according to the Preferred Reporting Items for Systematic Reviews and MetaAnalysis (PRISMA) statements checklist, including the publication of a PRISMA flow diagram.

Surveys were stratified as: (1) in-person (face to face or telephone), (2) postal, (3) e-mail, (4) Web-based with an online questionnaire, and (5) a miscellaneous group with mixed-mode surveys. The number of survey requests sent to the subjects was classified as a single request or multiple requests. The response rate per country of origin was described for all countries with $\geq 3$ identified surveys; otherwise, surveys were described as part of a continent or as miscellaneous for collaborations between continents. The response rate per scientific journal was described for the 10 journals with the highest number of identified surveys, displaying journals with $\geq 5$ identified surveys.

To establish an initial reporting guideline for surveys in surgical education, reporting guidelines available in the literature were collected. The EQUATOR (Enhancing the QUAlity and Transparency Of health Research) network was used to select the SURGE and CHERRIES guidelines as the most appropriate reference guidelines [14]. The initial guideline was written in accordance with the points raised in the reports on good practices in survey research [5, 15-22]. The reporting guideline by Moher et al. [22] was used an example.

The data are presented as mean (SD). Groups were compared by Student $t$ test for differences between 2 groups, and one-way analysis of variance for differences between the 5 survey modes. $p<0.05$ was considered statistically significant. Statistical analyses were performed with $R$ : A Language and Environment for Statistical Computing v1.0.153 for Mac ( $R$ Foundation for Statistical Computing, Vienna, Austria), using the software $R$ Package "ggplot2."

\section{Results}

In total, 5,693 records were retrieved from the systematic literature search, with 312 surveys meeting the selection criteria. A detailed description of the inclusion and exclusion of records is provided as a PRISMA flow diagram (online suppl. Fig. 1; for all online suppl. material, see www.karger.com/doi/10.1159/000516125). Of the 


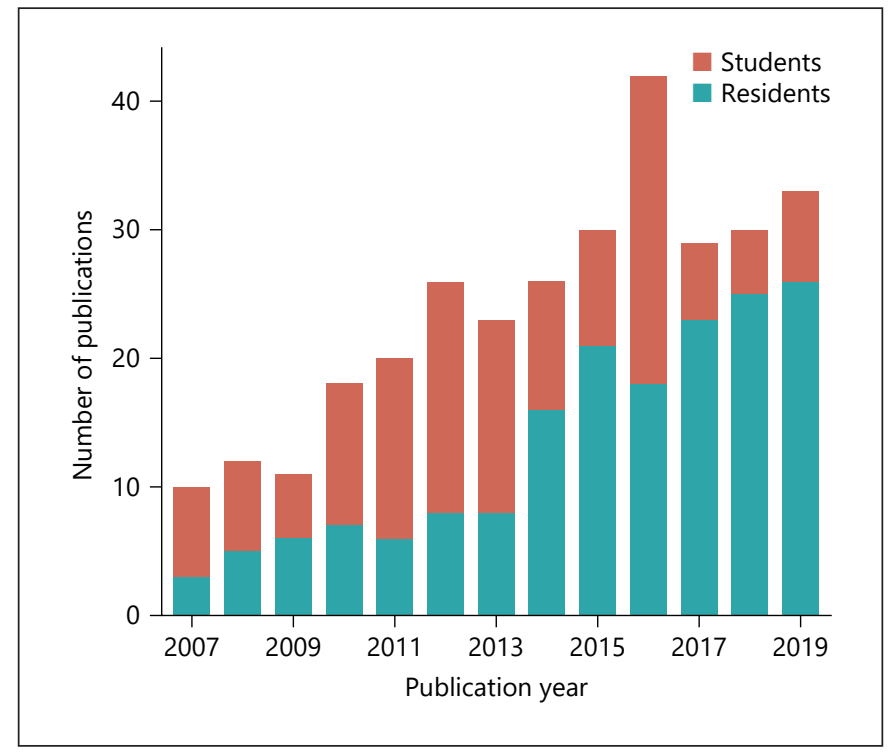

Fig. 1. Number of publications identified for each year, stratified for surgical residents and medical students, from 2007 to 2019.

identified surveys, 173 focused on surgical residents and 139 on medical students. One hundred and sixty-six (53.2\%) surveys were classified as Web-based, 89 (28.5\%) as in-person, $41(13.1 \%)$ as e-mail, and $16(5.1 \%)$ as postal mode. The majority of surveys was performed in the USA $(n=163,52.2 \%)$, followed by Canada $(n=32,10.2 \%)$, and the UK $(n=26,8.3 \%)$.

\section{Response Rates for Surgical Residents and Medical \\ Students}

The annual number of published surveys in surgical education increased from 10 in 2007 (3 surgical resident and 7 medical student surveys) to 33 in 2019 (26 and 7, respectively) (Fig. 1). The mean (SD) response rate for all 312 surveys was $61.6 \%(23.9 \%)$, with a mean of $55.7 \%$ (24.7\%) for surgical residents and 69.0\% (20.8\%) for medical students $(p<0.0001)$. When comparing the response rate between surgical residents and medical students for different modes of survey, the mean response rate was only statistically different for in-person surveys, with a mean of $73.5 \%(12.4 \%)$ and $81.3 \%(13.0 \%)$, respectively $(p=0.028$; Fig. 2).

\section{Response Variation for Modes of Survey and Repeated} Requests

A significant difference in response rates was observed between different survey modes $(p<0.0001)$, with in-person surveys resulting in the highest response rate (mean

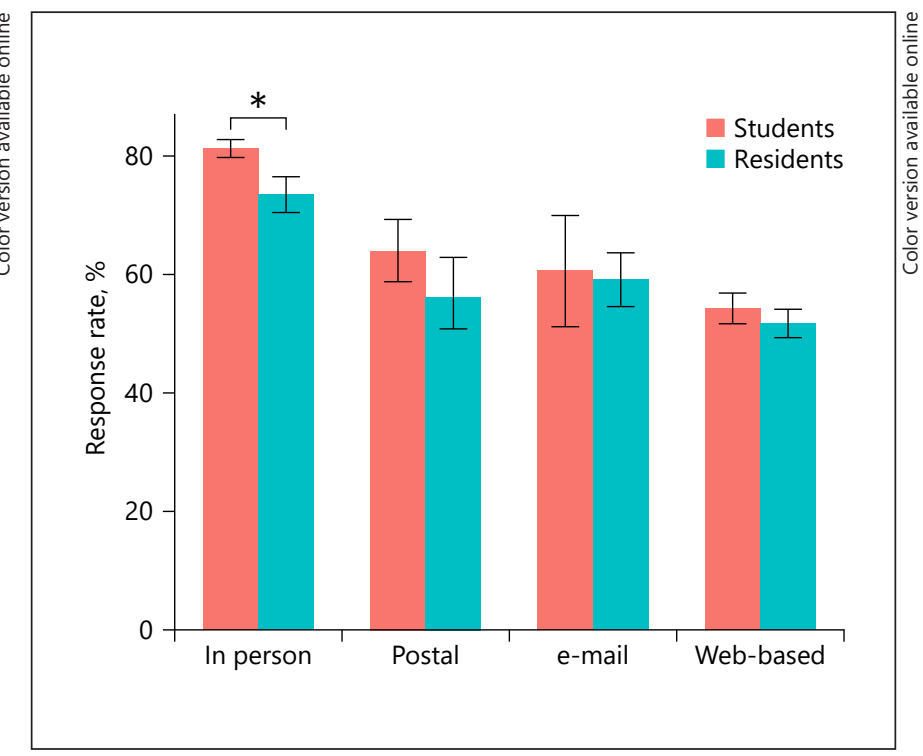

Fig. 2. Mean (SD) response rate for different modes of survey, stratified for surgical residents and medical students. $p$ value for Student $t$ test, with ${ }^{*} p=0.028$ indicating significance.

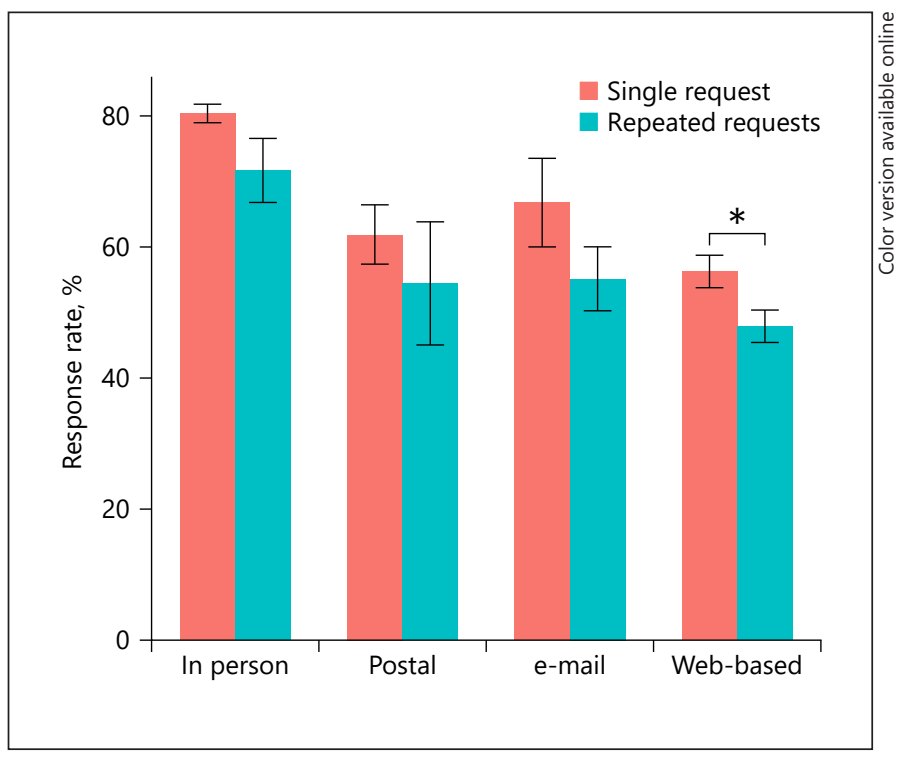

Fig. 3. Mean (SD) response rate for different modes of survey, stratified for single request and repeated requests surveys. $p$ value for Student $t$ test, with $* p=0.028$ indicating significance.

$79.8 \%(13.1 \%))$ and Web-based surveys in the lowest (mean 52.6\% (23.2\%)). The mean response rate was 59.4\% (25.7\%) for e-mail surveys and $59.1 \%(17.7 \%)$ for postal surveys. Lower response rates were observed for studies reporting multiple/repeated survey requests than those 
Fig. 4. Mean (SD) response rate per country or continent of origin.

Fig. 5. Mean (SD) response rate for the 10 journals with the highest number of identified surveys.
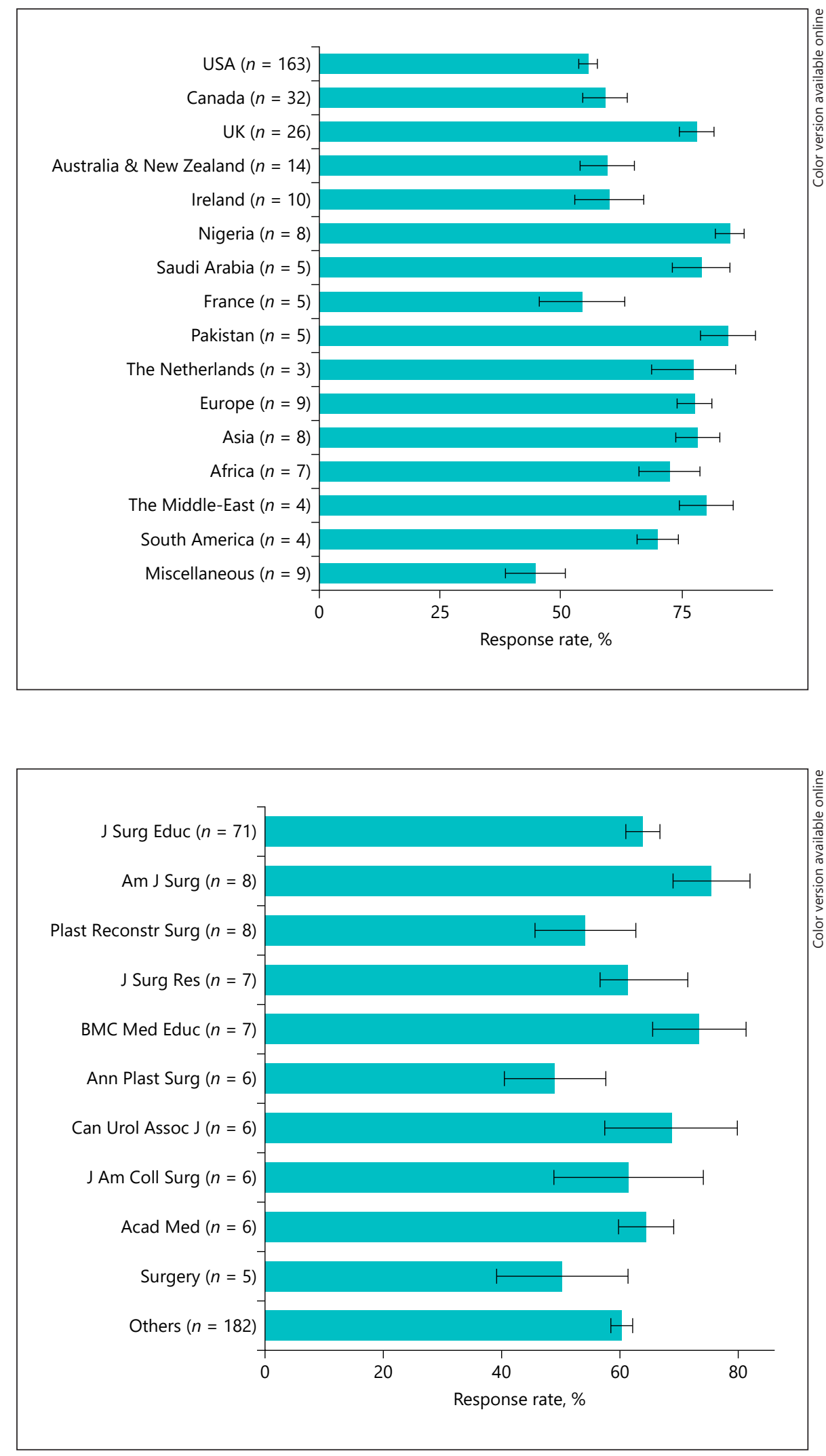
Table 1. Checklist of items to include when reporting a survey

\begin{tabular}{|c|c|c|}
\hline \multicolumn{3}{|l|}{ Title } \\
\hline & Title structure & 1. Identify the study as a survey and address the subjects of the survey \\
\hline \multicolumn{3}{|c|}{ Abstract } \\
\hline & Concise methodology & $\begin{array}{l}\text { 2. The subject group, mode of survey, study period, number of subjects approached, } \\
\text { and number of requests sent }\end{array}$ \\
\hline \multicolumn{3}{|c|}{ Introduction } \\
\hline & Rationale & 4. Clear description of the rationale, referring to important previous studies \\
\hline & Objectives & 5. Concise study aim, including the subject group and main research question \\
\hline \multicolumn{3}{|c|}{ Methods } \\
\hline & Subject group & 6. The specific subject group, including eligibility criteria \\
\hline & Survey items & $\begin{array}{l}\text { 10. A summary of the survey items, with information on reliability and validation, and } \\
\text { the full survey text as supplementary information }\end{array}$ \\
\hline & Missing data & 11. A description of missing data for each survey item \\
\hline & Data analysis & $\begin{array}{l}\text { 12. Analyses of qualitative and quantitative survey data, including the applied methods } \\
\text { for statistics }\end{array}$ \\
\hline \multicolumn{3}{|l|}{ Results } \\
\hline & General survey results & $\begin{array}{l}\text { 13. Number of subjects included, the total response rate, and response rate for each } \\
\text { request sent }\end{array}$ \\
\hline & (Non)respondents & 14. Subject characteristics of respondents and nonrespondents \\
\hline \multicolumn{3}{|c|}{ Discussion } \\
\hline & Reflection & 15. Critical reflection about the results and the available literature \\
\hline
\end{tabular}

reporting a single request, with a mean of $51.3 \%(22.3 \%)$ and $67.2 \%(22.9 \%)$, respectively $(p<0.0001)$. When comparing the difference between multiple and single requests for different modes of survey, the mean response rate was only statistically different for Web-based surveys, with a mean of $47.9 \%(21.2 \%)$ and $56.2 \%$ (24.2\%), respectively ( $p=0.019$; Fig. 3 ).

\section{Response Variation for Countries and Journals}

A wide variation in response rates was seen worldwide, with a mean response rate of $55.6(24.3 \%)$ in the USA, $59.0 \%$ (26.0\%) in Canada, and $78.0 \%(18.1 \%)$ in the UK. The highest mean response rate of $84.8 \%(8.4 \%)$ was seen in Nigeria and the lowest response rate in France with $54.3 \%$ (19.8\%) (Fig. 4). In Nigeria, all the surveys were in-person surveys; in France, all the surveys were Web-based (online suppl. Fig. 2). A variation was seen across the 10 journals with the highest number of identified surveys, with a mean response rate of $63.9 \%(24.6 \%)$ for the Journal of Surgical Education, 75.5\% (18.3\%) for the American Journal of Surgery, and 64.1\% (19.7\%) for the Journal of Surgical Research. The highest mean response rate was seen in the American Journal of Surgery and the lowest in the Annals of Plastic Surgery, with 49.1\% (21.0\%) (Fig. 5).

\section{Initial Reporting Guideline}

With 17 items that covered the key elements of a survey study manuscript, a clear and consistent presentation of the methodology and outcomes was generated (Table 1). The most important items of this guideline are the eligibility criteria for the selection of subjects, the mode of survey used, the survey items included, the number of subjects included, and the total response rate. 


\section{Discussion}

The yearly number of published surveys in surgical education has increased in the past 13 years, especially in surveys focusing on surgical residents. In-person surveys resulted in the highest response rate and Web-based surveys in the lowest response rate. Surgical residents responded less often to survey requests than medical students did, especially when the surveys were performed in person.

Previous studies evaluating response rates among different health care professionals showed a variety of outcomes. A review evaluating the response rate in doctors found that they have a lower response rate than patients (mean: 53.3 and 70.0\%, respectively [2]). Response rates in studies on surgeons, for example, are often very low (15\%), which could be explained by their busy workload and limited priority for participation [23]. Although medical students and residents are part of the health care system in terms of pursuing a medical career, their behavior in terms of survey participation cannot be compared to the behavior of doctors.

Our results are in agreement with a study on health profession residents, mostly medical students, that found a response rate of $71.3 \%$ (69.4\% for medical students) [12]. The relatively high response rate in surgical residents and medical students could be explained by the hierarchy they are subjected to in the health system, leading to a higher priority to participate [11]. We found a higher response rate for medical students than surgical residents, possibly explained by the combination of a higher rank in the hierarchy for residents and a busier work-schedule.

In our study, we found that in-person surveys resulted in the highest (79.8\%) and Web-based surveys in the lowest $(52.6 \%)$ response rate. These results are in line with previous studies $[3,16,24,25]$. However, because of the low response rates, such surveys can be subject to bias because of the self-selection of participants, the so-called "volunteer effect" [26]. To increase the response rate, Web-based surveys often use multiple requests for participation. We found that studies using repeated survey requests had lower response rates than those using a single request. This outcome is the opposite of previous studies, suggesting that multiple requests lead to higher response rates [9, 27]. According to the current guidelines, certain well-designed surveys achieve a high response rate on initial presentation [16]. When faced with a low response rate, authors are inclined to send additional requests. However, the factors that result in a low response rate in the first round will not be addressed only by sending repeat requests. This may explain why studies with repeated survey requests had a lower overall response rate. It is important to implement strategies to improve the response rate, and therefore decrease the risk of a nonresponse bias. This could be by implementing incentive-based interventions (that use money and design-based approaches), i.e., in-person surveys and user-friendly questionnaires that are not too long [28, 29].

A global variation in response rates was found. Surveys performed in Pakistan had the highest response rate $(84.8 \%)$ and those in France had the lowest (54.3\%). The response rate in the USA was also low (55.6\%). These differences can mostly explained by the differences in modes of survey (online suppl. Fig. 2). In nonwestern countries, like Nigeria, in-person or telephone surveys are preferred due to limited logistics and internet access [24]. The surveys in Pakistan and Nigeria, the countries with the highest response rate, were all conducted in person, the survey mode proven to lead to the highest response rate.

On the other hand, in France and the USA (the countries with the lowest response rates), the Web-based survey, i.e., the mode with the lowest response rate, was most often used. Another possible explanation could be cultural cross-country differences, like differences in individualism and collectivism [30,31]. However, the literature on cultural difference and survey outcomes is scarce, thus limiting conclusions on this matter.

Strategies to improve response rates and therefore the quality of surveys are often lacking. A systematic review of 100 Web-based and 100 non-Web-based surveys showed that many items of the Survey Reporting GuidelinE (SURGE) or the Checklist for Reporting Results of Internet E-Surveys (CHERRIES) guideline were not reported [20]. To provide a framework for surveys in surgical education, we compiled a 17-item initial reporting guideline to ensure clear and consistent presentation of the methodology and outcomes of survey studies in this field.

To our knowledge, this is the first systematic review on the response rates of surveys in surgical education. Strengths of this study include the systematic approach following the PRISMA reporting guideline and the relatively large number of survey studies included $(n=312)$. The main limitation of the study is the quality of the included records, with sparse information available about the methodology of the surveys performed. This limitation hampered a more elaborate analysis of the factors that influence response rates. The reporting guideline for surveys in surgical education can be considered as an initial step towards a broadly recognized guideline, which requires an expert review by means of a Delphi study [32].

In conclusion, this systematic review on surveys in surgical education demonstrated variations in response rates 
between different modes of survey, thus highlighting the importance of clear and consistent reporting of survey methodology.

\section{Statement of Ethics}

The paper is exempt from Ethics Committee approval since it does not include data on human subjects.

\section{Conflict of Interest Statement}

The authors have nothing to disclose.

\section{Funding Sources}

This research did not receive any specific grant from funding agencies in the public, commercial, or not-for-profit sectors.

\section{Author Contributions}

L.B.D. and S.B.: study design, data collection, statistical analysis, drafting the work, AND final approval. V.M.M.: study design, data collection, revising the work critically, final approval. J.K.: data collection, revising the work critically, AND final approval. J.F.M.L. and R.A.P.: study design, revising the work critically, and final approval.

\section{References}

1 Palmquist E, Ricard C, Chen L. Review of Surgical Education Research Trends in North America. J Surg Educ. 2019 Nov - Dec;76(6): 1476-83.

2 Meyer VM, Benjamens S, Moumni M El, et al. Global Overview of Response Rates in Patient and Health Care Professional Surveys in Surgery: A Systematic Review. Ann Surg. 2020, Online ahead of print.

3 Leung DY, Kember D. Comparability of data gathered from evaluation questionnaires on paper and through the internet. Res High Educ. 2005;46(5):571-91.

4 Gerbase MW, Germond M, Cerutti B, Vu NV, Baroffio A. How Many Responses Do We Need? Using Generalizability Analysis to Estimate Minimum Necessary Response Rates for Online Student Evaluations. Teach Learn Med. 2015;27(4):395-403.

5 Jones TL, Baxter MA, Khanduja V. A quick guide to survey research. Ann R Coll Surg Engl. 2013 Jan;95(1):5-7.

6 Sax LJ, Gilmartin SK, Bryant AN. Assessing response rates and nonresponse bias in web and paper surveys. Vol. 44, Research in Higher Education. Springer; 2003. p. 409-32.

7 Paolo AM, Bonaminio GA, Gibson C, Partridge $\mathrm{T}$, Kallail $\mathrm{K}$, Teaching and Learning in Medicine Response Rate Comparisons of EMail-and Mail-Distributed Student Evaluations Kallail (2000) Response Rate Comparisons of E-Mail-and Mail-Distributed Student Evaluations, Teaching and Learning in Response Rate Comparisons of E-Mail-and MailDistributed Student Evaluations. Medicine (Baltimore). 2009;12(2):81-4.

8 Phillips AW, Reddy S, Durning SJ. Improving response rates and evaluating nonresponse bias in surveys: AMEE Guide No. 102. Med Teach. 2016;38(3):217-28.

9 Brtnikova M, Crane LA, Allison MA, et al. A method for achieving high response rates in national surveys of U.S. primary care physicians. PLoS One. 2018;13(8):e0202755.

10 McColl E, Jacoby A, Thomas L, et al. Design and use of questionnaires: A review of best practice applicable to surveys of health service staff and patients. Health Technol Assess. 2001;5(31):1-256.
11 Gardeshi Z, Amini M, Nabeiei P. The perception of hidden curriculum among undergraduate medical students: a qualitative study. BMC Res Notes. 2018 May;11(1):271.

12 Phillips AW, Friedman BT, Utrankar A, Ta AQ, Reddy ST, Durning SJ. Surveys of health professions trainees: Prevalence, response rates, and predictive factors to guide researchers. Acad Med. 2017 Feb;92(2):222-8.

13 Bennett C, Khangura S, Brehaut JC, Graham ID, Moher D, Potter BK, et al. Reporting guidelines for survey research: an analysis of published guidance and reporting practices. PLoS Med. 2010 Aug;8(8):e1001069.

14 The EQUATOR network. Enhancing the QUAlity and Transparency Of health Research [Internet]. http://www.equator-network.org.

15 Grimshaw J. SURGE (The SUrvey Reporting GuidelinE) [Internet]. Guidelines for Reporting Health Research: A User's Manual. Oxford, UK: John Wiley \& Sons, Ltd; 2014 Aug.

16 Eysenbach G. Improving the quality of Web surveys: the Checklist for Reporting Results of Internet E-Surveys (CHERRIES). J Med Internet Res. 2004 Sep;6(3):e34.

17 Kelley K, Clark B, Brown V, Sitzia J. Good practice in the conduct and reporting of survey research. Int J Qual Health Care. 2003 Jun; 15(3):261-6.

18 Burns KE, Kho ME. How to assess a survey report: a guide for readers and peer reviewers. CMAJ. 2015 Apr;187(6):E198-205.

19 Li AH, Thomas SM, Farag A, Duffett M, Garg AX, Naylor KL. Quality of survey reporting in nephrology journals: a methodologic review. Clin J Am Soc Nephrol. 2014 Dec;9(12):208994.

20 Turk T, Elhady MT, Rashed S, Abdelkhalek M, Nasef SA, Khallaf AM, et al. Quality of reporting web-based and non-web-based survey studies: what authors, reviewers and consumers should consider. PLoS One. 2018 Jun; 13(6):e0194239.

21 Jones D, Story D, Clavisi O, Jones R, Peyton P. An introductory guide to survey research in anaesthesia. Anaesth Intensive Care. 2006 Apr; 34(2):245-53.

22 Moher D, Schulz KF, Simera I, Altman DG Guidance for developers of health research re- porting guidelines. PLoS Med. 2010 Feb; 7(2):e1000217.

23 Sprague S, Quigley L, Bhandari M. Survey design in orthopaedic surgery: getting surgeons to respond. J Bone Joint Surg Am. 2009 May; 91(3 Suppl 3):27-34.

24 Blumenberg C, Barros AJ. Response rate differences between web and alternative data collection methods for public health research: a systematic review of the literature. Int J Public Health. 2018 Jul;63(6):765-73.

25 Cook DA, Wittich CM, Daniels WL, West CP, Harris AM, Beebe TJ. Incentive and Reminder Strategies to Improve Response Rate for Internet-Based Physician Surveys: A Randomized Experiment. J Med Internet Res. 2016 Sep; 18(9):e244.

26 Eysenbach G, Wyatt J. Using the Internet for surveys and health research. J Med Internet Res. 2002 Apr-Nov;4(2):E13.

27 Burgess C, Nicholas J, Gulliford M. Impact of an electronic, computer-delivered questionnaire, with or without postal reminders, on survey response rate in primary care. J Epidemiol Community Health. 2012 Jul;66(7):6634.

28 VanGeest JB, Johnson TP, Welch VL. Methodologies for improving response rates in surveys of physicians: a systematic review. Eval Health Prof. 2007 Dec;30(4):303-21.

29 Edwards PJ, Roberts I, Clarke MJ, Diguiseppi C, Wentz R, Kwan I, et al. Methods to increase response to postal and electronic questionnaires. Cochrane Database Syst Rev. 2009 Jul;(3):MR000008.

30 Nicoletti C, Peracchi F. Survey response and survey characteristics: microlevel evidence from the European Community Household Panel. J R Stat Soc A. 2005;168(4):763-81.

31 Sykes LL, Walker RL, Ngwakongnwi E, Quan $\mathrm{H}$. A systematic literature review on response rates across racial and ethnic populations. Can J Public Health. 2010 May-Jun;101(3): 213-9.

32 Banno M, Tsujimoto Y, Kataoka Y. Reporting quality of the Delphi technique in reporting guidelines: a protocol for a systematic analysis of the EQUATOR Network Library. BM] Open. 2019 Apr;9(4):e024942. 